\title{
Research of HMCVT Speed Change Law Based on Optimal Productivity
}

\author{
Mingzhu Zhang ${ }^{\mathrm{a}}$, Dongyang Bai ${ }^{\mathrm{b}}$, Quansheng Wang ${ }^{\mathrm{c}}$ \\ School of Mechtronics Engineering, Henan University of Science and Technology, Luoyang \\ 471003, China

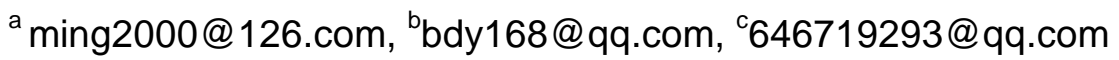

Keywords: Speed change law; tractive power; hydro-mechanical continuosly variable transmission; tractor

\begin{abstract}
To get the optimal productivity, the speed change law is studied for the 400 horsepower wheeled tractor equipped with hydro-mechanical continuosly variable transmission (HMCVT). The tractive power are proposed as the evaluation indexes of the producitvity of the tractor. Based on the load resistance and vehicle speed, an optimization process of optimal productivity is established, and according to the optimization process, the matching engine speed and HMCVT speed ratio are calculated. for any load resistance and vehicle speed which are within the range of maximum value, the tractor can get optimal productivity by adjusting the trasmission speed ratio and engine speed to change as the values calculated. The research results show that the speed change law made in accordance with the optimized calculation can make the tractor obtain optimal productivity compaired with the initial value under the condition of any load resistance and the maximum speed range. the slip rate has an significant influence on tractive power, for 400 horse power tractor, controlling the load resistance within $60 \mathrm{kN}$ is good for getting higher productivity.
\end{abstract}

\section{Introduction}

The HMCVT has advantages of hydraulic drive and mechanical drive ${ }^{[1]}$, and its application are becoming more and more widely. These oversea studies for HMCVT are initially used in military vehicles and heavy trucks, the studies are foucsed on the speed-changing control strategy, such as shifting control strategy, steering control and comprehensive control, etc ${ }^{[2]}$. Domestic reasearch for HMCVT has focused on the transission theory, speed change law, fuel economy and control simulation and so on ${ }^{[3]}$. As the agricultural machinery for tractors, to obtain a higher productivity has always been the pursuing goals for tractor producers.

The application of HMCVT in tractors has made a big progress on the traction performance, fuel economy, and promoted the agricultural production. However, the domestic reaches for tractors equipped with HMCVT are mainly focused on dynamic performance, fuel economy, less on the productivity. Thus, the productivity research is of great significance. This article takes the 400 horsepower wheeled tractor as the research object, studied the speed change law based on the optimal productivity of tractors with HMCVT.

\section{Evaluation index of productivity}

The tractive power $W_{T}$ directly influences the tractor's productivity, it directly reflects the production power acting on the tractor hook ${ }^{[4]}$. The tractor's tractive power usually refers to the power acting on the hook, it is the output of engine power which needs to remove the trasmission power loss and the rolling friction loss on the draft implements. Thus there are:

$$
\begin{aligned}
& W_{T}=F_{T} v \\
& F_{T}=F_{q}-F_{f} \\
& v=\pi(1-s) n_{e} i_{b} r_{q} / 30 i_{z}
\end{aligned}
$$


Where, $F_{T}$ is the tractive power on the hook, $\mathrm{N} ; F_{q}$ is the tractor driving force, $\mathrm{N} ; F_{f}$ is the tracor's rolling resistance, $\mathrm{N} ; v$ is the tractor's velocity, $\mathrm{m} / \mathrm{s} ; s$ is the slip rate of the driving wheel; $r_{q}$ is the radius of the driving wheel, $\mathrm{m} ; n_{e}$ is the engine revolution speed, $\mathrm{r} / \mathrm{min} ; i_{b}$ is the transmission speed ratio ${ }^{[5]}$.

According to the experimental data, we can draw the tractive power characteristic curve of the 400 horsepower tractor when the engine are working in the rated speed condition, as shown in figure 1.

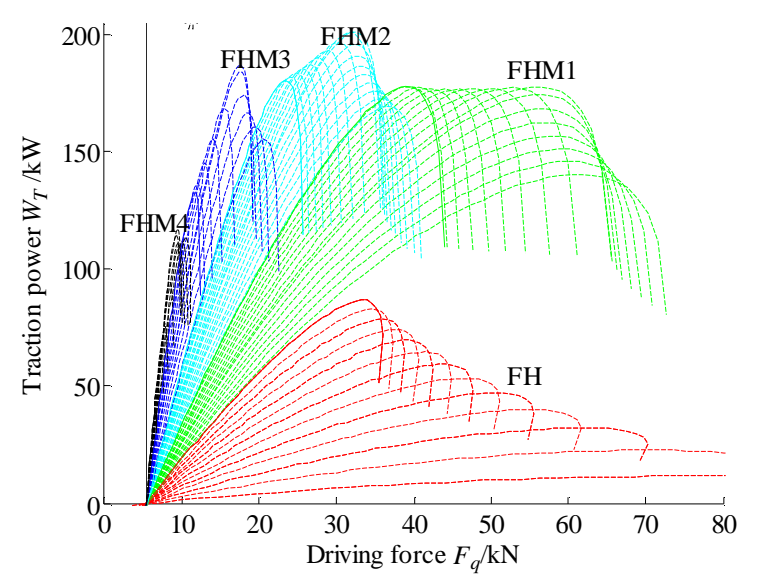

Figure 1 Tractive power characteristic curve

As can be seen from figure 1: when the HMCVT works at the pure hydraulic section $(\mathrm{FH})$, the tractor is in the starting stage. For the low efficiency properties of hydraulic transmission, the tractor's tractive power is at a lower level. While it can significantly increase the stability and comfort performance. When the HMCVT switch from pure hydraulic section to hydro-mechanical section(FHM1-FHM4), the tractive power gets a bigger increase, and it stabilizes at higher levels during a large range of driving force $(20-60 \mathrm{kN})$. But the maximum tractive power changes obviously with the change of driving force, and it cannot stabilize at the maximum position. This is not so good for the agricultural production which is always pursuiting a higher production. So the research of the HMCVT speed change law with the target of getting the optimal tractive power is of great significance.

\section{The HMCVT speed change law}

When traction operation is taking place, the tractor's productivity is mainly reflected by the traction performance, its basic requirement of stepless regulation is that: under any traction resistance, the tractor can all output the maximum tractive power. This article takes the tractive power as the productivity evaluation criteria to formulate the speed change law of HMCVT.

By formula (1) and (2), there are:

$$
W_{T}=\left(F_{q}-F_{f}\right) v
$$

and

$$
\begin{aligned}
& F_{q}=\frac{\eta_{b} \eta_{z} i_{z} T_{e}}{r_{q} i_{b}} \\
& F_{f}=\mu m g
\end{aligned}
$$

Where, $\mu$ is the rolling resistance coefficient; $m$ is the tractor mass, $\mathrm{kg}$.

By formula (4), (5) and (6), there is:

$$
W_{T}=\left(\eta_{z} i_{z} \eta_{b} T_{e} / r_{q} i_{b}-\mu m g\right) * \pi(1-s) r_{q} n_{e} i_{b} / 30 i_{z}
$$

The total efficiency of central drive and final drive is determined by the design parameters of the tractor, they can generally be regarded as quantify. For the particular soil, the rolling resistance coefficient can also be seen as quantify. Under the condition of the same ground, $s$ can generally be 
regarded as the function of the driving force $F_{q}$, changing in a wide range with the shift of driving force [6]. The transmission efficiency is the fuction of $i_{b}, n_{e}$ and $T_{e}$. By formula (7), the tractive power is influenced by the slip ratio, the transmission speed change ratio, the transmission efficiency and the engine speed position. Therefor, the tractor can output maximum tractive power by adjusting the transmission's speed ratio and the engine's speed position in control system of transmission binary adjustment, in order to meet the requirements of the optimal productivity.

For any load resistance, to ensure the tractor can output the optimal productivity within the maximum driving force determined by the slip rate is the requirement of the optimal productivity speed change law. Accordingly, $W_{T}$ should be the largest under arbitrary load resistance. The transmission correspondingly has a best speed ratio with each engine speed position meeting the $W_{T}$ is the largest. Thus, the tractor can work at the position of maximum tractive power by adjusting the transmission's speed ratio and the engine's speed position, to get the optimal productivity.

For any load resistance, we can determine the working point of the tractor according to the matching actual tractive force and velocity. To determine the working point of the HMCVT $\left(T_{b}, \omega_{b}\right)$ by formula (8) and (9); Then, according to $\left(T_{b}, \omega_{b}\right)$, caculating the HMCVT speed ratio $i_{b}$ and the engine speed $n_{e}$ that make the $W_{T}$ be the largest; Finally, controlling the HMCVT and engine to automatically adjust $i_{b}$ and $n_{e}$, to achieve the higest productivity speed change law, and this is the HMCVT's higest productivity speed change rule for tractors.

The conversion relationship of the HMCVT output characteristic is as follows:

$$
\begin{gathered}
T_{b}=\frac{T_{q}}{i_{z} \eta_{z}}=\frac{\left(F_{T}+\mu m g\right) r_{q}}{i_{z} \eta_{z}} \\
\omega_{b}=i_{z} \omega_{q}=\frac{i_{z} v}{r_{q}(1-s)}
\end{gathered}
$$

Due to solving the speed ratio $i_{b}$ that satisfies the largest $W_{T}$ is much more complex, so the optimum transmission speed ratio $i_{b m}$ that satisfies the largest $W_{T}$ can be calculated in advance, and stored in the memory unit of the control system ${ }^{[7]}$.

\section{Optimization calculation for the optimal productivity}

For any load resistance and vehicle speed, there always are corresponding HMCVT speed ratio and engine speed can make the $W_{T}$ be the largest. Because of the nonlinear function about $n_{e}, T_{e}, i_{b}$, the calculation belongs to the problem of reverse solution. That is very complex, so we need to calculate the HMCVT efficiency before the speed change rule is made and all the possible conditions should be considered.

(1) optimization variables

The optimization variables include the transmission speed ratio $i_{b}$, the engine speed $n_{e}$, and the engine torque $T_{e}$.

$$
X=\left[i_{b}, T_{e}, n_{e}\right]
$$

(2) Optimization objective

The optimization target is to make the $W_{T}$ be optimal.

$$
\operatorname{Maxf}(X)=\max \left[W_{T}\right]
$$

For $\eta_{s}$, fitting out the calculating formula according to the test dates, $\eta_{s}=1-s / 100$.

$$
s=1.1865+0.0079 * \exp \left(0.1190 * \mathrm{~F}_{T}\right)-1.2131 * \exp \left(-0.1190 * \mathrm{~F}_{T}\right)
$$

(3) Constraint conditions: to limit the engine speed and torque range and the speed-changing ratio of the HMCVT. 
$\left\{\begin{array}{l}1300 \leq n_{e} \leq 2300 \\ 0 \leq T_{e} \leq 1200 \\ 0 \leq\left|i_{b}\right| \leq 2.1152\end{array}\right.$

(4) Power constraint: the power outputted by the HMCVT should be limited within the maximum value of the engine $\left(N_{\text {emax }}\right)$.

$$
T_{b} * \omega_{b} \leq N_{e \max }
$$

The optimization calculation of speed ratio is obtained by using the calculating process shown in figure 2 .

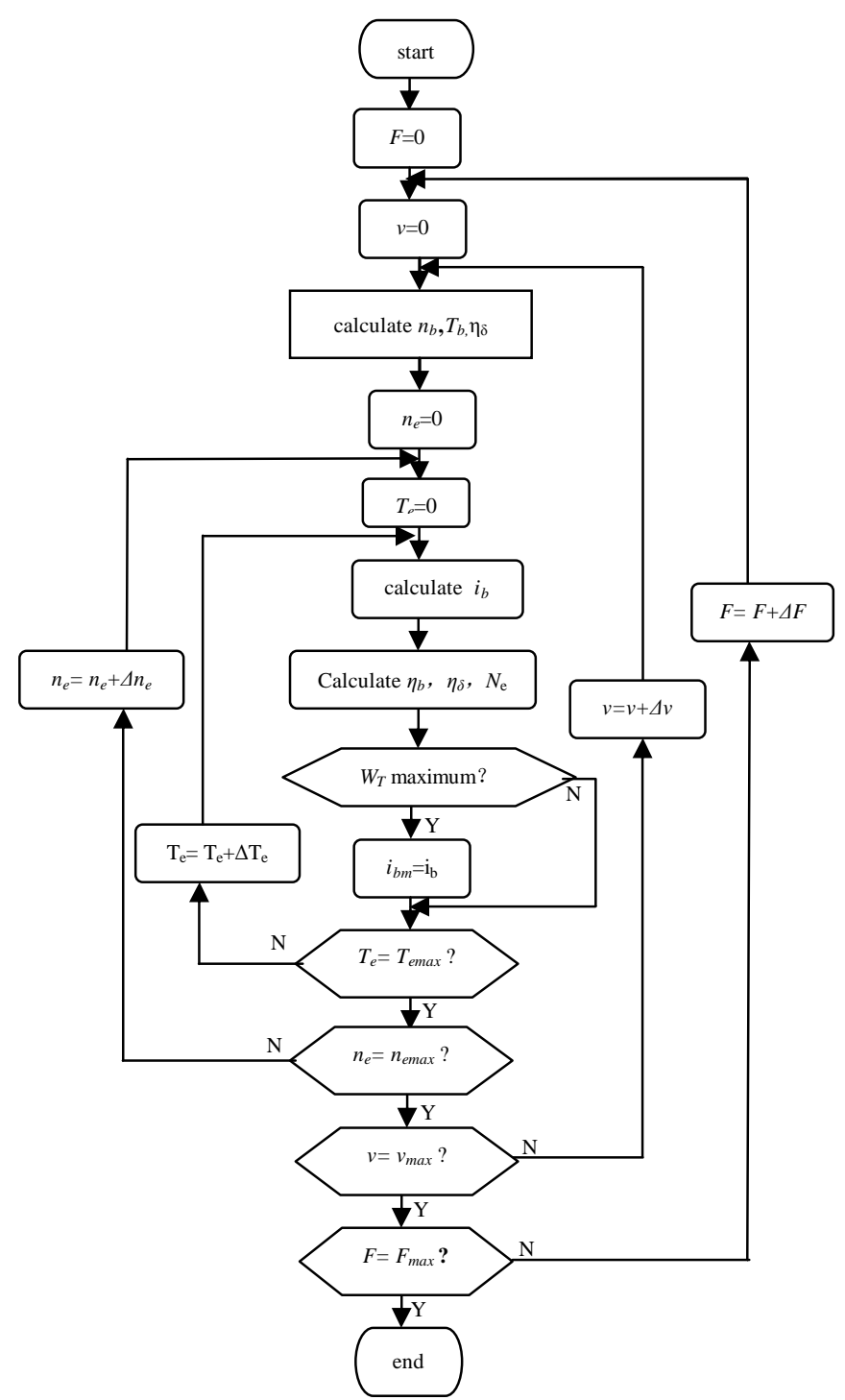

Figure 2 Calculation flow chart

The calculated optimal speed ratio and engine speed distribution according to the calculating process shown in figure 2 are as shown in the figures below.

Analysis of the optimization results:

(1)Figure 3(a) is the optimal transmission speed ratio distribution diagram after optimization, as can be seen from figure 3(a): within the maximum driving force, for any load resistance, at different vehicle speeds, there is a corresponding transmission speed ratio meeting the largest tractive power. For the same vehicle speed, and for different load resistance, the rangeability of the optimal speed ratio is not big, but with the increase of the load resistance, the slip rate also increases. In order to keep the same 
vehicle speed, the speed ratio must be increased. The cocking-up on the top right corner of figure 3(a) is caused by the increase of slip rate.

(2) Figure 3(b) is the engine speed distribution diagram after optimization, as can be seen from figure 4: when the speed is $0 \mathrm{~km} / \mathrm{h}$, the tractor is in the state of idling, the matching engine speed is determined by the design. With the increase of the speed and the change of the load, the engine speed also increases until reaching the working speed. Then, the tractor speed can be adjusted by the transmission ratio for the changing load. The engine speed has corresponding fluctuation for the diffient efficiency before and after the grade-changing.

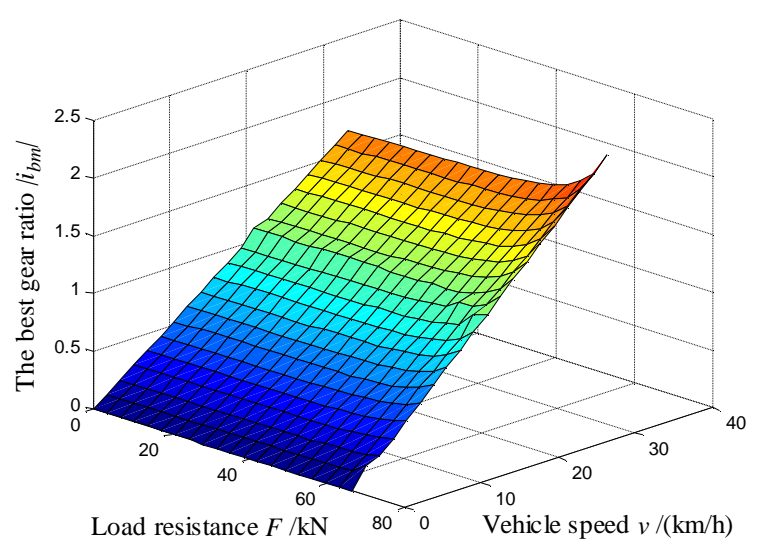

(a)

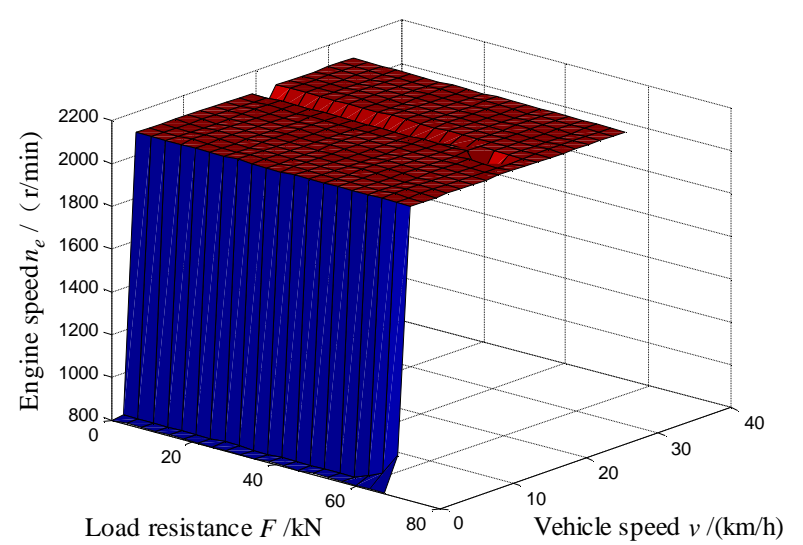

(b)

Figure 3 (a) The optimal speed ratio distribution after optimization

Figure 3(b) The engine speed matching with the optimal tractive power

(3) Figure 4 is the tractive power distribution diagram after optimization, as can be seen from figure 4: within the maximum driving force,for any load resistance and vehicle speed,the tractor equipped with HMCVT can output larger tractive power, the area of big numerical tractive power is larger(contrast to figure 1). This shows that the productivity performance of the whole area is good. In the figure, the transverse fluctuation is caused by the change of the efficiency of HMCVT. When the vehicle speed is definited, with the continuous increase of load resistance, the slip rate also increase. After the load resistance exceeding $60 \mathrm{kN}$, the tractive power reduces due to the increasing slip rate.

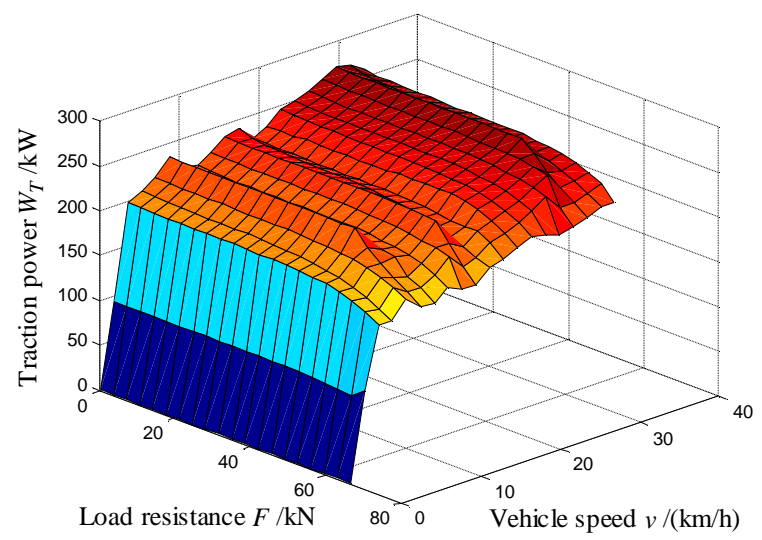

Figure 4 The tractive power after optimization

So the driving force should be controled in the area of big tractive power shown in figre 4 to reduce the influence of slip loss.

(4) The optimal HMCVT speed ratio and engine speed after optimization can be stored in the memory unit of the drive system controller as the target HMCVT speed ratio and the target engine speed. To make the tractor work in the status of outputting the higest productivity through adjusting 
the transmission speed ratio and engin speed, this is the speed change law of the HMCVT based on the higest productivity.

\section{Conclusions}

(1) Tractive power is proposed as the index of productivity evaluation. In order to get the optimal productivity, an optimizing calculation for the engine speed and the HMCVT's speed ratio is established, According to the calculation process, the transmission speed change law has been made. The optimization results show that the calculating process is reasonable, the tractor can obtain optimal tractive power.

(2) The study provides a theoretical basis for the establishment of control strategy based on the variable speed governing position and the transmission speed ratio. Slip rate has an outstanding influence for the tractive power, for the 400 horsepower tractors, controlling the load resistance within $60 \mathrm{kN}$ is good for getting optimal productivity.

\section{Acknowledgements}

This paper is financially supported by the National Natural Science Foundation of China( Grant No.51375145).

\section{References}

[1] Xu Liyou, Zhou Zhili, et al. The design of tractor hydro-mechanical continuously variable transmission[J]. Transactions of the Chinese Society for Agricultural Machinery, 2006, 37(7): 5-8.

[2] Kimjczyk J. Continuously variable hydromechanical transmission for commercial vehicle by simulation studies[J]. SAE paper 840873, 1984.

[3] Hao Yunzhi, Sun Dongye, Lin Yupei, et al. The whole optimization control strategy of the continuously variable transmission system[J]. JOURNAL OF MECHANICAL ENGINEERING, 2013, 49(12): 84-91.

[4] Zhang Mingzhu, The control strategy research for tractor multistage hydro-mechanical continuously variable transmission[D]. Xi An: Xi'an University of Technology, 2007.

[5] Chen Lihui, Zhou Zhili, et al. The speed ratio control of tractor continuously variable transmission[J]. Journal of Henan University of Science and Technology: Natural Science, 2007, $28(2)$.

[6] Zhuang Jide, Calculation automobile terramechanics[M]. Bei Jing: CHINA MACHINE PRESS, 2002: 2-8.

[7] Zhang Yingjun, The control strategy research for tractor hydro-mechanical continuously variable transmission driving system[D]. Wu Han: Wuhan University of Technology, 2007. 